\title{
Spatio-spectral phase-matching in broadband soliton mode- locked lasers
}

\author{
Shih-Hsuan Chia ${ }^{1}$, Li-Jin Chen ${ }^{2}$, and Franz X. Kärtner ${ }^{1,2}$ \\ ${ }^{1}$ Center for Free-Electron Laser Science, DESY and Department of Physics, University of Hamburg, Notkestraße 85, \\ D-22607 Hamburg, Germany \\ ${ }^{2}$ Department of Electrical Engineering and Computer Science and Research Laboratory of Electronics, Massachusetts Institute of Technology, 77 \\ Massachusetts Avenue, Cambridge, Massachusetts 02139, USA \\ shih-hsuan.chia@desy.de
}

\begin{abstract}
A spatio-spectral phase-matching theory is introduced. It is used to improve broadband modelocking of a Ti:sapphire laser with a tunable enhancement of $>15 \mathrm{~dB}$ at long wavelengths while maintaining a good beam profile.

OCIS codes: (140.4050) Mode-locked lasers; (310.6845) Thin film devices and applications; (320.7090) Ultrafast lasers
\end{abstract}

\section{Introduction}

Ultrashort pulse lasers continue to have tremendous impact in science and technology. Especially passively modelocked lasers assisted by the Kerr nonlinearity are widely-employed in various broadband systems [1]. Moreover, self-phase modulation (SPM) due to intracavity materials and the interplay with cavity dispersion, which is often engineered by dispersion-compensating mirrors (e.g. double chirped mirrors (DCMs), and specially shaped output couplers (OCs) make it possible to obtain laser output spectra broader than the gain bandwidth of the gain medium [2-6]. Therefore, with an ultrabroadband gain medium, such as Ti:sapphire, octave-spanning spectra have been demonstrated using DCM pairs (DCMPs) [2] and a suitable OC [3-4]. The generated spectral wings outside the gain bandwidth have great importance in enabling frequency comb generation [3] and sub-two-cycle optical pulses [5] directly from the oscillator, which benefits many applications such as optical metrology and seeding of optical parametric amplifiers for attosecond science [7].

Despite the great experimental accomplishment of few-cycle Ti:sapphire lasers, broadband spatiotemporal characteristics of Kerr-lens mode-locked (KLM) lasers still fail to be precisely described and explained. Especially the spectral wings were often considered as non-resonant waves that occur in an uncontrollable manner with poor beam quality [6,8]. Therefore, performing a reliable analysis and further optimization for such lasers to achieve broadband spectra and good beam profile are still mostly relying on experimental observations. In fact, the wings and the ripple structures on the spectra can be explained by a general phase-matching process between a soliton-like wave and a dispersive wave. In this paper, we discuss the spatio-spectral phase-matching process in broadband soliton mode-locked lasers.

\section{Theory}

In a mode-locked laser in the soliton regime, the circulating pulse only gains a fixed amount of nonlinear phase and does not change its shape after each round-trip. However, unlike a real soliton which always maintains the pulse shape during the propagation due to the balance of SPM and group delay dispersion (GDD), the shape and the spectrum of the pulse travelling inside the laser cavity change dramatically within each round-trip. Also, in addition to SPM and GDD, the soliton-like mode-locked pulse experiences loss, gain, and higher order dispersion during propagation. Since the impact of loss, gain, and higher order dispersion per round-trip are usually small in steady state, they can be treated as perturbations to a dispersion-managed soliton whose dynamics is still dominated only by SPM and GDD. These perturbations lead to radiation of dispersive waves. The low intensity dispersive wave propagates around the cavity with only linear effects like dispersion and diffraction. When the phase contributed by the linear effects equals the nonlinear phase experienced by the soliton wave, the newly generated and existing dispersive waves will coherently add up, resulting in resonantly enhanced peaks in the spectrum. The phasematching process described here is similar to several previous works, Kelly sideband generation [9], third-orderdispersion-induced resonance [10], and fiber-optic Cherenkov radiation [11].

In the solid-state laser cavity, the Kerr effect not only induces coupling between longitudinal modes via SPM but also transverse modes via self-focusing. The dispersive wave from soliton perturbation at each wavelength can be viewed as a superposition of transverse modes. In the linear propagation regime, higher-order modes experience more Gouy phase than lower-order modes, which leads to a mode-dependent phase-matching condition. Here we assume that the soliton is mainly in the fundamental transverse mode due to the mode matching with the pump beam. Therefore, the phase-matching condition is satisfied when the phase of the soliton-like wave with respect to 
the center wavelength per round trip equals to the phase of the linear dispersive wave. That is, by requiring that the nonlinear phase $\phi_{N L}$ is matched to the linear phase contributed by the cavity dispersion $\phi_{D}$ and the mode-dependent round-trip Gouy phase $\phi_{\text {Gouy,RT }}$, one can write a general phase-matching condition for Laguerre-Gaussian beam assuming radial symmetry with radial index $p$ :

$$
\phi_{N L}+\phi_{\text {Gouy }, R T}+2 m \pi=\phi_{D}+(2 p+1) \phi_{\text {Gouy, } R T}
$$

Commonly observed spectral peaks in the spectral wings [5] can be predicted by the phase-matching theory. To analyze the mode structures and the spectral peaks, we numerically setup a laser cavity using the dispersion and reflectivity calculated from the real mirror designs [12]. The cavity is operated at a nearly dispersion-free point around the center wavelength. The stimulated spectrum and the overall round-trip cold cavity phase near $650 \mathrm{~nm}$ are shown in Fig. 1. The simulation result shows a good agreement of the spatio-spectral enhancement with the phasematching condition.

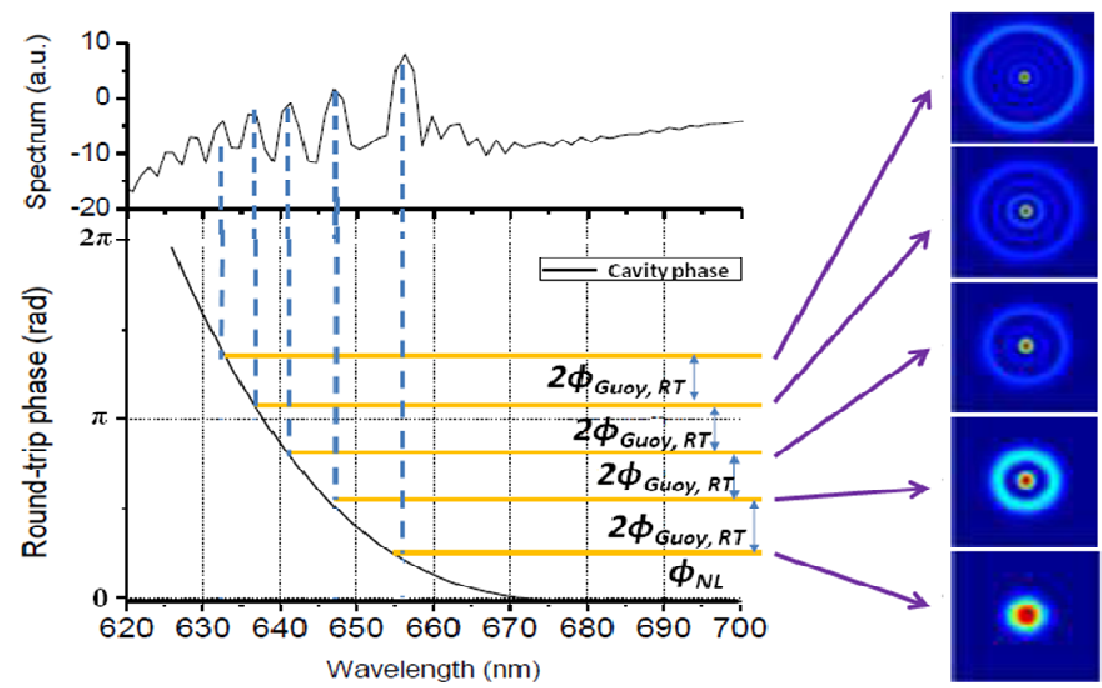

Fig. 1. The resonant peaks near $650 \mathrm{~nm}$ of the simulated spectrum. (bottom) cavity round trip phase $\phi_{D}$ due to dispersion. The color plots on the right show the beam profile corresponding to the phase-matched wavelengths..

\section{Optimization of laser spectrum and experimental demonstration}

To demonstrate the spectral enhancement, we add an artificial phase profile to the intracavity roundtrip phase that provides phase-matching for the wavelength range around $1140 \mathrm{~nm}$ : the cold cavity phase increases above $900 \mathrm{~nm}$ linearly and the dispersion is perfectly compensated below $900 \mathrm{~nm}$. The simulated spectrum with the artificial phase feature shows a significant enhancement as high as $15 \mathrm{~dB}$ when compared with the case with zero net cavity dispersion over the whole wavelength range [13]. In addition, the overall spectrum results in a 4.6fs transformlimited pulse width, which is even shorter than for the zero dispersion case, which would result in a 5.5fs transformlimited pulse width. Therefore, we designed and fabricated a 5\% PMOC based on a multilayer dielectric coating. Multilayer dielectric coatings have large design freedom and the presented technique provides a general solution which can be adapted to lasers with vastly different gain media. The red curves in Fig. 2(a) show the calculated group delay (GD) and the induced net phase of the PMOC. By decreasing the cavity dispersion by the equivalent of $0.83 \mathrm{~mm}$ optical path length of $\mathrm{BaF}_{2}$, the design phase can be achieved with a phase ramping up from $900 \mathrm{~nm}$ to $1200 \mathrm{~nm}$, see Fig. 2(a). The measurement results shown in blue are in good agreement with the design curves shown in red.

The PMOC was tested in a commercial $85 \mathrm{MHz}$ ultrabroadband Ti:sapphire laser (Thorlabs Octavius-85M) where DCMPs are used to compensate the material dispersion including two adjustable $\mathrm{BaF}_{2}$ wedges for dispersion fine tuning. Fig. 2(b) shows the experimental spectra. Since the intracavity enhancement process is based on the coherent build-up between the already existing dispersive wave in the cavity around the phase-matched wavelength and the newly-generated dispersive wave after each round-trip, the process becomes weaker when the cavity loss or output-coupling is high. Therefore, the output coupling coefficient should not be too high. Furthermore, the low output coupling window should stretch over the phase-matched wavelength range to maintain the phase-matching enhancement while supporting good beam quality. The red curve in Fig. 2(b) shows only a 15dB difference between 
the central maximum spectral content and the spectral wings around the phase-matched wavelength of $1140 \mathrm{~nm}$, which is much higher than the current state-of-art case even when using only a $1 \%$ OC of $\sim 25 \mathrm{~dB}$ [3]. According to the phase-matching theory, the ripples on the spectra can be explained by the phase ripples induced by the intracavity DCMPs. In addition, the phase ramp with an almost linear slope makes it possible to control the phasematched peak by slightly changing the cavity dispersion without compromising the stability and spectral bandwidth. By changing the insertion depth of the $\mathrm{BaF}_{2}$ wedge, the phase-matched peak can be continuously tuned from $0.97 \mu \mathrm{m}$ to $1.06 \mu \mathrm{m}$. In Fig. 2(b), the optical path difference of $\mathrm{BaF}_{2}$ between the red spectrum and the purple one is only $\sim 0.2 \mathrm{~mm}$, corresponding to $7.6 \mathrm{fs}^{2}$ of GDD at $0.8 \mu \mathrm{m}$. Thus, we can obtain stable operation without compromising the stability and spectrum within the gain bandwidth much, which is ideal to optimize seeding of ytterbrium-doped pump laser systems used for parametric amplification and frequency conversion of the Ti:Sapphire spectrum.

(a)

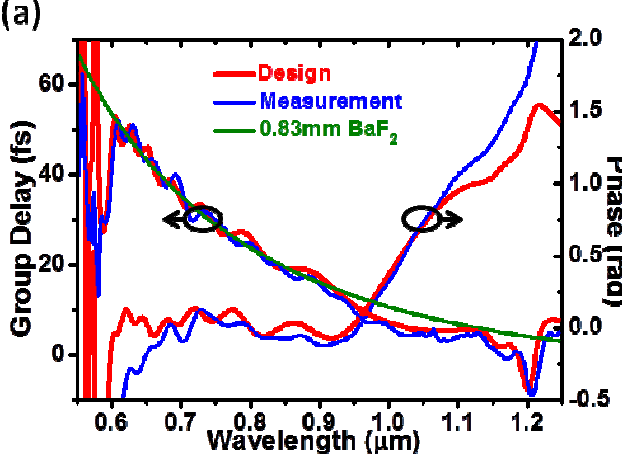

(b)

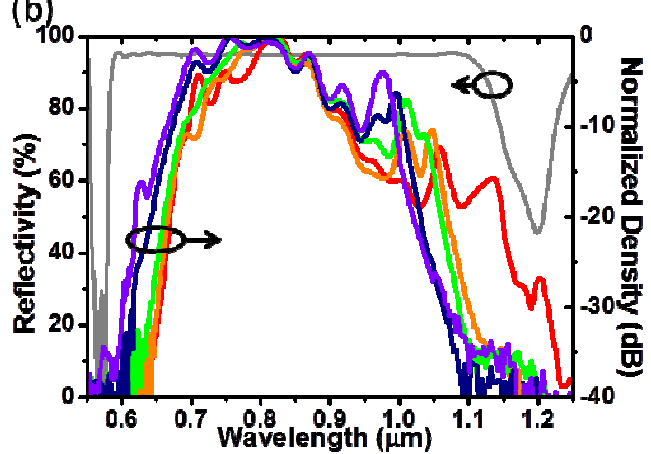

Fig. 2. (a) Design GD and corresponding net induced phase (red curves), measured GD and corresponding net induced phase (blue curves), and GD of $0.83 \mathrm{~mm} \mathrm{BaF}_{2}$. (b) The reflectivity of the PMOC (gray curve) and the experimental spectra. The spectral peak can be continuously tuned from the red curve to the purple one by increasing the insertion depth of $\mathrm{BaF}_{2}$ wedges.

Optimization strategies based on a better understanding of cavity dispersion control and intracavity mirror designs for broadband lasers will be discussed: the cold cavity phase can be engineered to enhance the spectral density especially in the spectral wings where phase-matching is achieved; the corresponding output coupling wavelength range should be expanded to cover the matched wavelengths for better enhancement and beam quality. The tunable spectral wings in our design potentially benefits several applications as seeding of optical parametric amplifiers and the stabilization of the direct Ti:sapphire frequency combs. Even-broader spectra from current stateof-art lasers could thus be possible for pursuing shorter pulse generation.

\section{References}

[1] E. P. Ippen, "Principles of passive mode locking," Appl. Phys. B 58, 159 (1994).

[2] F. X. Kärtner, U. Morgner, R. Ell, T. Schibli, J. G. Fujimoto, E. P. Ippen, V. Scheuer, G. Angelow, and T. Tschudi, "Ultrabroadband doublechirped mirror pairs for generation of octave spectra," Opt. Soc. Am. B 18, 882 (2001)..

[3] L. Matos, D. Kleppner, O. Kuzucu, T. R. Schibli, J. Kim, E. P. Ippen, and F. X. Kärtner, "Direct frequency comb generation from an octavespanning, prismless Ti:sapphire laser," Opt. Lett. 29, 1683 (2004).

[4] L.-J. Chen, M. Y. Sander, and F. X. Kärtner, "Kerr-lens mode locking with minimum nonlinearity using gain-matched output couplers," Opt. Lett. 35, 2916 (2010).

[5] H. M. Crespo, J. R. Birge, E. L. Falcao-Filho, M. Y. Sander, A. Benedick, and F. X. Kärtner, "Non-intrusive phase-stabilization of sub-twocycle pulses from a prismless octave-spanning Ti:sapphire Laser," Opt. Lett. 33, 833 (2008).

[6] T. M. Fortier, D. J. Jones, and S. T. Cundiff, “Phase stabilization of an octave-spanning Ti:sapphire laser,” Opt. Lett. 28, 2198 (2003).

[7]. S.-W. Huang, G. Cirmi, J. Moses, K.-H. Hong, S. Bhardwaj, J. R. Birge, L.-J. Chen, E. Li, B. J. Eggleton, G. Cerullo, and F. X. Kärtner, "High-energy pulse synthesis with sub-cycle waveform control for strong-field physics," Nat. Photonics 5, 475 (2011).

[8] S. T. Cundiff, W. H. Knox, E. P. Ippen, and H. A. Haus, "Frequency-dependent mode size in broadband Kerr-lens mode locking," Opt. Lett. $21,662(1996)$

[9] P. F. Curley, Ch. Spielmann, T. Brabec, F. Krausz, E. Wintner, and A. J. Schmidt.,’Operation of a femtosecond Ti:sapphire solitary laser in the vicinity of zero group-delay dispersion," Opt. Lett., 18, 54-56 (1993)

[10] S. M. Kelly, "Characteristic sideband instability of periodically amplified average soliton,” Electron. Lett. 28 (8), 806 (1992)

[11] G. Chang, L.-J. Chen, and F. X. Kärtner, "Fiber-optic Cherenkov radiation in the few-cycle regime,” Opt. Express, 19, 6635 (2011)

[12] L.-J. Chen, C.-J. Lai, and F. X. Kärtner, "The Role of Plasma Formation in Mode-locking of Few-cycle Ti:sapphire Lasers: A Spatiotemporal Model," CWR5 in the digest of CLEO, Baltimore, MD (2011).

[13] S.-H. Chia, L.-J. Chen, and F. X. Kärtner, "Broadband mode-locking with phase-matched output couplers," CTh1H5 in the digest of CLEO, San Jose, CA (2013). 